Chapter 11

\title{
Trachoma and Inclusion Conjunctivitis
}

\author{
Udo Ubani \\ Additional information is available at the end of the chapter \\ http://dx.doi.org/10.5772/53861
}

\section{Introduction}

Trachoma is the leading infectious cause of preventable blindness in the world today and occurs as mesoendemic, endemic, or hyperendemic disease. The distribution is primarily in tropical developing countries of the world including North and sub-Saharan Africa, the Middle East, and the Northern Indian subcontinent. The disease has also been reported in Southeast Asia and specific regions of Central and South America, Australia, and the South Pacific Islands. Of the more than 600 million people afflicted, approximately 150 million have visual deficits, and 12 million are predicted to be blind by the year 2020 .

\section{Clinical features}

\subsection{Symptoms}

In the absence of secondary infection, symptoms are minimal and include mild foreign body sensation in the eyes, occasional lacrimation, slight stickiness of the lids and scanty mucoid discharge. In the presence of secondary infection symptoms of acute mucopurulent conjunctivitis develop.

\subsection{Signs}

\subsubsection{Conjunctival}

1. Congestion of upper tarsal and forniceal conjunctiva

2. Conjunctival follicles of 0.2 to $2 \mathrm{~mm}$ in diameter and are commonly seen on upper tarsal conjunctiva and fornix, but may also be present in the lower fornix, plica semilunaris 
and caruncle. Sometimes, follicles may be seen on the bulbar conjunctiva. Follicles are formed due to the scattered aggregation of lymphocytes and other cells in the adenoid layer. Central part of each follicle is made up of mononuclear histiocytes, few lymphocytes and large multinucleated cells called Leber cells. The cortical part is made up of zone of lymphocytes showing active proliferation. Blood vessels are present in the most peripheral part. In later stages signs of necrosis are also seen.

3. Papillary hyperplasia: Papillae are reddish, flat topped raised areas which give red and velvety appearance to the tarsal conjunctiva. Each papilla consists of central core of numerous dilated blood vessels surrounded by lymphocytes and covered by hypertrophic epithelium.

4. Conjunctival scarring may be irregular, star-shaped or linear. Linear scar present in the sulcus subtarsalis is called Arlt's line.

5. Concretions may be formed due to accumulation of dead epithedial cells and inspissated mucus in the depressions called glands of Henle.

\subsubsection{Corneal}

1. Superficial Keratitis may be present in the upper part.

2. Herbert follicles refer to typical follicles present in the limbal area. These are histologically similar to conjunctival follicles.

3. Pannus which is an infilteration of the cornea associated with vascularization, can develop at any point along the limbal margin but is most pronounced at the superior limbal margin. The vessels are superficial and lie between epithelium and Bowman's membrane; and later on the Bowman's membrane is destroyed.

Pannus may be progressive (infiltration of cornea is ahead of vascularization) or regressive also termed pannus siecus (vessels extend a short distance beyond the area of infiltration).

4. Corneal ulcer may sometimes develop at the advantage of pannus. Such ulcers are usually shallow which may become chronic and indolent.

5. Herbert pits are the oval or circular pitted scars, left after healing of Herbert follicles in the limbal area.

6. Corneal opacity may be present in the upper part. It may even extend down and involve the papillary area. It is the end result of trachomatous corneal lesions.

\subsection{Differential diagnosis}

1. Presence of large multinucleated Leber cells and signs of necosis differentiate trachoma follicles from Adenovirus follicles conjunctivitis.

The distribution of follicles in trachoma is mainly on upper palpebral conjunctiva and fornix. Sometimes, follicles may be seen on the bulbar conjunctiva. 
The corneal involvement of the disease includes lymphoid follicle formation at the limbus. This is a characteristic feature of trachoma and can lead to the development of Herbert's pit.

Associated signs such as papillae and pannus are characteristic of trachoma.

2. Trachoma with predominant papillae is differentiated from palpebral form of spring catarrh as follows:

Papillae are large in size and usually there is typical cobble-stone arrangement in spring catarrh.

The $\mathrm{pH}$ of tears is usually alkaline in spring catarrh, while in trachoma it is acidic.

Discharge is ropy in spring catarrh

The corneal involvement of the disease includes lymphoid follicle formation at the limbus. This is a characteristic feature of trachoma and can lead to the development of Herbert's pit. In trachoma, there may be associated follicles and pannus.

\section{Sequelae of trachoma}

1. Sequelae in the lids may be trichisis, entropion, tylosis (thickening of lid margin), ptosis, madarosis and ankylophearon.

2. Conjunctival sequelae include concretions, pseudocyst, xerosis and symblepharon.

3. Corneal sequelae may be corneal opacity, ectasia, corneal xerosis and total corneal pannus (blinding sequelae).

4. Other sequelae may be chronic daccryocystitis, and chronic dacryoadenitis.

The only complication of trachoma is corneal ulcer and it occurs due to rubbing by concretions, or trichiasis with superimposed bacterial infection.

\section{Classification}

Trachoma has always been an important blinding disease under consideration of WHO and thus many attempts have been to streamline its clinical profile. The latest classification suggested by WHO in 1987 with the acrimony FISTO is as follows:

1. TF: Trachomatous inflammation-follicular is the stage of active trachoma with predominantly follicular inflammation. To diagnose this stage at least five or more follicles (each $0.5 \mathrm{~mm}$ or more in diameter) must be present on the upper tarsal conjunctiva. Further, the deep tarsal vessels should be visible through the follicles and papillae. 
2. TI: Trachomatous inflammation-intense is diagnosed when pronounced inflammatory thickening of the upper tarsal conjunctiva obscures more than half of the normal deep tarsal vessels.

3. TS: Trachomatous scarring is diagnosed by the presence of scarring in the tarsal conjunctiva. These scars are easily visible as white, bands or sheets (fibrosis) in the tarsal conjunctiva.

4. TT: Trachomatous trichiasis is labeled when at least one eyelash rubs the eyeball. Evidence of recent removal of intured eyelashes should also be graded as trachomatous trichiasis.

5. CO: Corneal opacity is labeled when easily visible corneal opacity is present over the pupil. This sign refers to corneal scarring that is so dense that at least part of pupil margin is blurred when seen through the opacity. The definition is intended to detect corneal opacities that cause significant visual impairment (less than 6/18).

\section{The causative organism Chlamydia trachomatis}

\subsection{Taxanomy}

Chlamydial organisms were historically referred to as Bedsonia or Miyagawanella and were initially thought to be protozoa. Because of their small size and the problems encountered with propagation, they were subsequently thought to be viruses. In the 1960s they were classified as bacteria because Chlamydia express proteins (e.g., lipopolysaccharides) that are functionally analogous to other bacteria, divide by binary fission, are inhibited by antibacterial drugs, contain ribosomes, and are structurally and morphologically similar to gram-negative bacteria. However, Chlamydia are only distantly related to other eubacterial orders based on phylogenies of ribosomal ribonucleic acid (rRNA) gene sequences. Chlamydiae comprise their own order, Chlamydiales; a single family, Chlamydiaceae; and one genus, Chlamydia. The genus Chlamydia is comprised of four known species: C. trachomatis, C. psittaci, C. pneumoniae, and Chlamydia pecorum.

Chlamydia trachomatis is made up of three biologic variants or biovars: trachoma, lymphogranuloma venereum (LGV), and the rodent biovar that includes the mouse pneumonitis (MoPn) and hamster strains. There is $87 \%$ to $99 \%$ deoxyribonucleic acid (DNA) homology among the human strains and biovars of C. trachomatis but only $30 \%$ homology for the rodent strains.

With the exception of the rodent strains, C. trachomatis is currently known to infect only humans. The infections in humans include the conjunctiva and lower and upper genital tracts, including the rectum and lymphatics that drain the perineum. These infections are caused by the 19 currently recognized serologic variants or serovars (defined by monoclonal and polyclonal antibodies that react to epitopes on the major outer membrane protein MOMP of C. trachomatis.) of the trachoma and LGV biovars. 

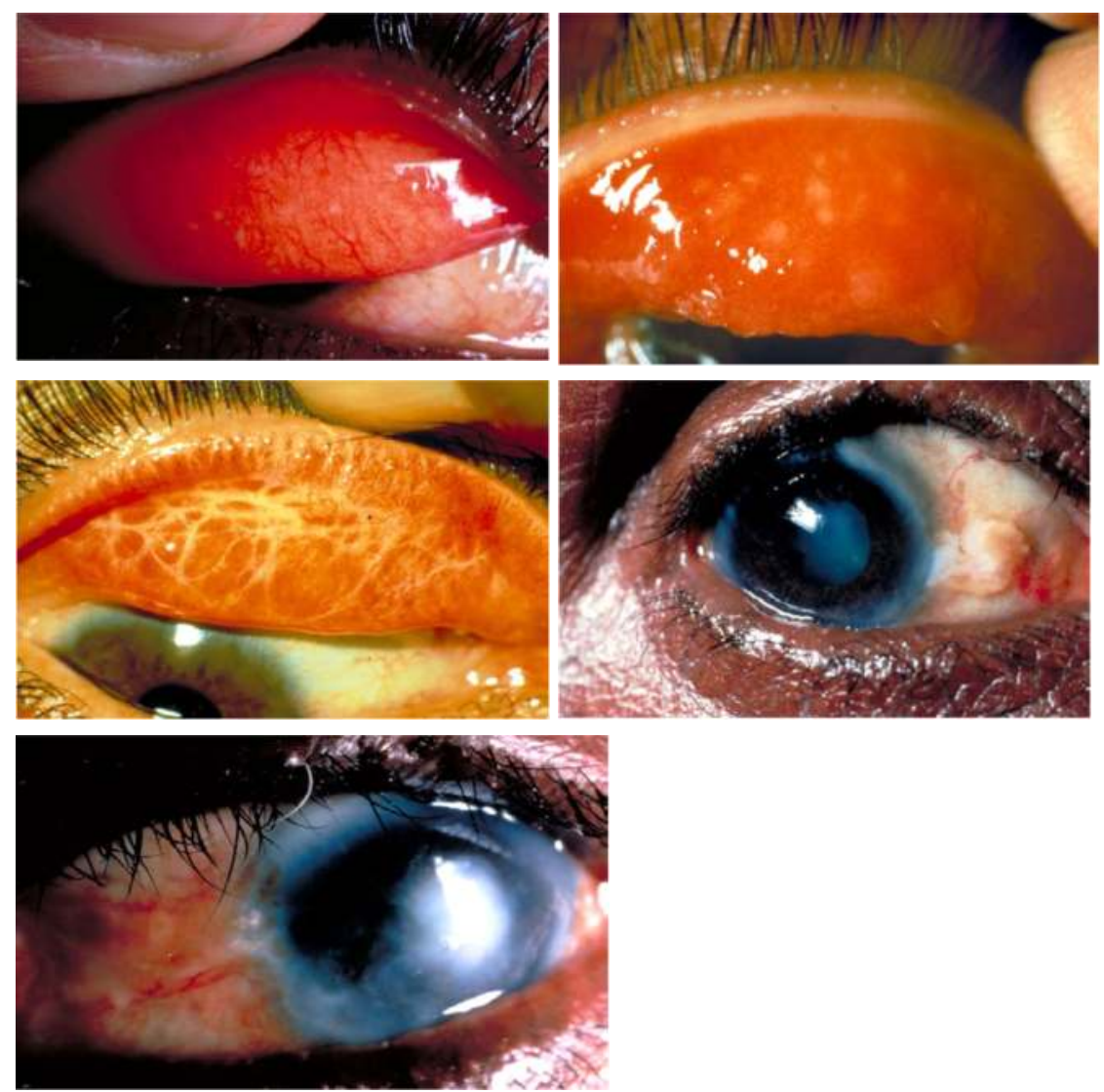

Figure 1. Top left: Trachomatous Inflammation - Follicular Presence of follicles on the flat surface of the upper tarsalconjunctiva.Top right: Trachomatous Inflammation - Intense With enlarged vascular papillae marked inflammatory thickening of the upper tarsal conjunctiva obscures the deep conjunctival vessels. Middle left: Trachomatous Scarring The scar (white/yellow) lines form a 'network' of fibrous scarring in the tarsal conjunctiva. Middle right: Trachomatous Trichiasis There is evidence of one or more eyelashes rubbing on the eyeball.Bbottom: Corneal Opacity The patient has significantly reduced vision due to corneal scarring.

Serotyping has distinguished these serovars into different serogroups or classes: B class (serovars B, Ba, D, Da, E, L2, and L2a), intermediate class (serovars F and G), and C class (serovars A, C, H, I, Ia, J, Ja, K, Ka, L1, and L3). Serovars A through K and Ba, Da, $\mathrm{Ia}, \mathrm{Ja}$, and $\mathrm{Ka}$ were previously referred to as trachoma-inclusion conjunctivitis (TRIC) strains. Trachoma is primarily caused by serovars A, B, Ba, and C, whereas adult and neonatal inclusion conjunctivitis are caused by serovars $\mathrm{B}$ or $\mathrm{Ba}$, $\mathrm{D}$ through $\mathrm{K}$, $\mathrm{Da}$, Ia, Ja, Ka, L1, L2, L2a, and L3, which are the sexually transmitted strains of the organism. The LGV serovars tend to cause more severe disease and can invade regional lymphatics, whereas the non-LGV serovars are currently known to infect epithelial cells at ocular, respiratory, rectal, and genital mucosal surfaces. 
Serotyping has been the most widely accepted technique for classifying C. trachomatis organisms. However, within the last decade, a new technique has been developed based on sequencing of ompA and is referred to as ompA genotyping. (ompA was previously called omp1, but the nomenclature has changed to be consistent with that of other bacteria.) This latter technique has been and continues to be invaluable for evaluating the molecular epidemiology, disease pathogenesis, and transmission dynamics of chlamydiae for STD and trachoma populations.

\subsection{Chlamydia trachomatis development cycle}

Chlamydia trachomatis are obligate intracellular parasites that are unable to synthesize their own energy (ATP) and are completely dependent on their host for energy. It has a unique biphasic developmental cycle not found in any other bacteria. There is the elementary body (EB) is the infectious form (spore-like particle) that posses a rigid outer membrane that bind to receptors on host cells and initiate infection. and the reticulate body (RB), which is the metabolically active form. Once the EB comes in contact with susceptible epithelial cells, it attaches by divalent cations and polycations, using heparin sulfate as a bridge between receptors on the EB and the cell surface. The EB is taken up into a phagosome by receptor-mediated endocytosis. There is ineffectual lysosomal fusion with the endophagosome because of their rigid outer membrane, and hence intracellular survival is insured.

A vacuole encloses the elementary body and the bacterium is now a reticulate body. Reticulate bodies obtain their energy by sending forth "straw-like" structures into the host cell cytoplasm. It can then replicate itself through binary fission. After division, the reticulate body becomes the elementary body. Anywhere from 100 to 1000 EBs can be produced per infected cell. In many cases, the cell ruptures and dies releasing the infectious progeny, but the cell can also extrude the inclusion body by a process of exocytosis and is released trough reverse endocytosis

\section{Diagnosis}

\subsection{Clinical}

A presumptive diagnosis of trachoma can be made based on clinical features, especially in an area where trachoma is considered to be present. The following signs are important indicators for trachoma, and at least two must be present in person diagnosed: follicles in the upper tarsal conjunctiva, limbal follicles or their sequelae, Herbert's pits, typical conjunctival scarring, and pannus. These signs along with conjunctival detection of C. trachomatis in the laboratory confirm the presence of endemic or hyperendemic trachoma in the respective area. 


\subsection{Laboratory}

\subsubsection{Cytology}

The conjunctiva is swabbed with a Dacron or cotton swab, and the smear is made by rolling the swab over a clean glass slide. Alternatively, the swab can be placed in a special transport media for the respective diagnostic test. Epithelial cells that are clearly separated and the presence of PMNs, lymphocytes, plasma cells, or Leber cells (giant macrophages that contain phagocytosed material) can denote an adequate sampling of the conjunctiva. The degree of inflammation and bacterial superinfection can also be appreciated from these smears. Thus, although not diagnostic for chlamydiae, these findings are suggestive of trachoma. Giemsa stain is inexpensive, and the test is easy to perform, which makes it attractive for developing countries where trachoma is endemic or hyperendemic. However, the sensitivity is only about $60 \%$ and, thus, should not be used in areas of low endemicity. With this stain, the inclusion body is visualized as a basophilic, stippled inclusion in contrast to the dark blue to purple color of the cell. However, other entities can also stain similarly: These include goblet cells, bacteria, keratin, nuclear extrusions, and eosinophilic granules. Lugol's iodine stains the glycogen-containing inclusion of $\mathrm{C}$. trachomatis. It imparts a dark yellow-brown color to the inclusion but is infrequently used, because it is insensitive. Commercially available fluorescent [fluorescein isothiocyanate (FITC)] conjugated monoclonal antibodies against the MOMP, which is species-specific for $\mathrm{C}$. trachomatis, or the LPS, which is genus-specific for Chlamydia, are used in this test. The EBs are stained an apple green color and are visualized as extracellular round dots. The sensitivity for this test is approximately $80 \%$ to $90 \%$.

\subsubsection{Tissue culture}

Although the intracellular inclusions of trachoma were first identified by Halberstaedter and von Prowazek4 in 1907, the actual organisms were finally cultured in 1957 by using chick embryos. Today, tissue culture has supplanted the use of eggs, which has made isolation of chlamydiae more widely available, although it is still only performed in specialized reference laboratories. Tissue culture remains the gold standard for $\mathrm{C}$. trachomatis identification but is not $100 \%$ sensitive, probably because of the difficulty in maintaining a cold chain $\left(4^{\circ} \mathrm{C}\right.$ for no longer than 24 hours and then $\left.-70^{\circ} \mathrm{C}\right)$ from the field site to a specialized reference lab where the culture will actually be performed. Also, because some viability is lost on freezing and some of the trachoma serovars are more difficult to propagate, culture requires technical expertise, can take 3 to 6 days for results, and is very expensive. Many different cell lines are now available for culture, including HeLa and McCoy cells. Additional passages in tissue culture can increase the positive rate but have other drawbacks, including a delay in the reporting time of the results. Fluorescein-conjugated antibodies are used to detect the inclusions in cell culture, which are visualized as intracytoplasmic ovoid, round, or irregularly shaped inclusions. This stain imparts a fluorescing, apple green color to the inclusion body that stands out against the dark red cells that have been counterstained with 
Evans blue. Peroxidase-conjugated monoclonal antibodies are also available for the detection of chlamydial inclusion bodies.

\subsubsection{Antigen detection}

ELISA or enzyme immunoassay (EIA) commercial assays are available to detect chlamydiae, but the sensitivity is only $70 \%$ to $85 \%$. However, these assays can be cost effective compared with other commercially available tests such as the DNA detection assays. These tests detect the EB via polyclonal or monoclonal antibodies directed against the genus-specific chlamydial LPS. The antibodies are conjugated with an enzyme that reacts with a substrate to produce a change in color that can be detected by a specific wavelength in a spectrophotometer. One advantage is that a 96-well format can be used to process multiple samples at one time. Less technical expertise is required than for the above-mentioned tests. Another advantage is that the kits contain a confirmatory test.

\subsubsection{DNA detection}

The commercial LCR and PCR tests are the most recent assays to be developed for detecting Chlamydia. Primers that are specific for the organism anneal to the complementary strand of DNA after denaturation. This target DNA is usually the plasmid, which is only present in C. trachomatis and C. psittaci species. LCR amplifies a signal that occurs when the primers hybridize with the plasmid DNA. In PCR, the actual DNA is amplified after hybridization. Both tests can be used in a 96-well format in which 92 to 94 samples can be assayed at one time. Both products are detected by spectrophotometers that are set at specific wavelengths for the particular assay. An advantage to the commercial PCR test is that an internal control plate can be run in parallel with the chlamydial detection plate to identify which samples have inhibitors. Those samples that contain inhibitors can then be run by in-house PCR assays that employ a DNA purification protocol that removes the inhibitors.

Chlamydial DNA can also be detected by commercially available hybridization probes. These also hybridize with complementary plasmid or ompA DNA. The sample is usually a swab of the conjunctiva that has been applied to a special filter paper immediately after the sample has been obtained from the patient. Occasionally, DNA is extracted from a swab that has been placed in a special collection media and then is applied to a filter. In both cases, the filter is what is probed. The advantage of this technique is that the filter paper that contains the samples can be stored at room temperature under field conditions and transported back to the lab at a convenient time, without the necessity of a cold chain. The sensitivity of the probes is $70 \%$ to $90 \%$.

\subsubsection{Serology}

There are two serologic tests for Chlamydia: the microimmunofluorescent (MIF) test and the complement fixation (CF) test. However, neither is specific for the organism because patient sera can cross react with different serovars and species and may represent current or previous sexually transmitted infection as opposed to conjunctival infection. The highest antibod- 
ies detected in the assay, however, are usually found against the initial infecting serovar, even on subsequent infection. This concept is referred to as original antigenic sin. Furthermore, ocular chlamydial infections tend to be chronic and endemic. Thus, these assays cannot be used to diagnose active infection, although occasionally MIF has been used for epidemiologic studies. They are also only available in reference laboratories.

The CF test is the older of the two and detects group-reactive antigen on C. psittaci and C. trachomatis. This test can be used for diagnosing ocular infections resulting from LGV or C. psittaci. The MIF test employs EBs representing C. trachomatis serovars and usually one or two strains of C. pneumoniae and C. psittaci. Sera, tears, and other bodily fluids can be used in this assay. The fluids are serially diluted and reacted against the EBs that have been applied and fixed to a slide in groups of dots. A FITC conjugated antihuman IgM, IgG, IgA or secretory IgA antibody is used as the secondary antibody to detect antigen-antibody binding. The slides are screened under fluorescent microscopy for fluorescing EBs that represents the respective serovar or species. Serum IgM and IgG antibodies appear around 2- to 3-weeks postinfection and persist for 4 to 8 weeks, although the IgG antibodies persist for much longer. Occasionally, IgM titers can rise again with reinfection or relapse of infection. Approximately $80 \%$ of children in trachoma endemic areas and $90 \%$ of adults with inclusion conjunctivitis will have detectable MIF antibodies. About half of the population in trachoma endemic areas will have both serum and tear antibodies, and the titers are directly proportional to the severity of disease and to the presence of chlamydial organisms in the conjunctiva. In one study in Tunisia, $80 \%$ of children with severe disease, $31 \%$ with moderate disease, and $17 \%$ with mild disease had tear antibodies to chlamydiae. Although the highest titers are usually against the infecting serovar in ocular infections, the MIF test can be used only for a diagnosis of active infection in neonates not in older children or adults. Neonates acquire IgG antibodies from their mothers, and when they develop conjunctivitis, these titers usually do not change during the course of the ocular infection. Most infected neonates do develop a small rise in serum IgM antibodies, usually less than 1:32, which persists for a few weeks.

\section{Predisposing factors}

Children are considered the primary source of infection because they usually become easily infected from close contacts during frequent play and within small, crowded households. However, female caregivers can also serve as an important reservoir in which the infection is passed to them and then back to their children. Thus, children younger than 10 years are at greatest risk for infection and reinfection.

Young women of childbearing age and other female caregivers are reported to have infection rates that range from $5 \%$ to $10 \%$. These infections likely represent transmission within the household from and to children. Adult women develop more severe disease and sequelae than their male counterparts; repeat infection is considered an important factor in disease progression to trichiasis. 
Other factors are low socioeconomic status, poor facial hygiene and lack of water. Flies have historically been considered vectors for transmission in Africa. However, data from recent studies do not support the theory. In one study, fluorescein was used to stain the secretions in the eyes of children. Within 15 to 30 minutes, the legs and bodies of the flies were also stained with fluorescein. Eye-seeking flies such as Musca sorbens have been shown to land on the eyes of multiple children as was reported in a study in Africa, but it is not clear how many infectious EBs can be carried on the flies, how long they are viable, and whether the inoculum is sufficient to cause infection. In The Gambia, of 395 flies captured from the eyes of C. trachomatis-infected children, only two were positive by PCR and could not be confirmed. It is certainly possible that flies carry other bacteria from eye to eye, which might promote inflammatory disease and trachoma. Indeed, in many trachoma endemic countries, there are seasonal outbreaks of conjunctivitis resulting from multiple bacterial species including Haemophilus influenzae, Haemophilus aegyptius, Streptococcus pneumoniae, Neisseria meningitidis, N. gonorrhoeae, and Moraxella spp. These infections may actually precede periods of increased trachoma prevalence rates. In a study in Tunisia, moderate to severe trachoma was found significantly more often among children with bacterial coinfections. Furthermore, pathogenic and nonpathogenic bacteria commonly colonize children who reside in trachoma areas. Coinfection of $\mathrm{C}$. trachomatis with these bacteria may be one mechanism that is important for promoting severe inflammation, which results in conjunctival scarring and corneal vascularization years later.

\section{Management}

Management of trachoma involve curative as well as control measures. The World Health Assembly has resolved to eliminate blinding trachoma by the year 2020. To this, the Global Alliance for the Elimination of Blinding Trachoma (GET2020) was formed in 1998. Control activities focus on the implementation of the SAFE strategy, surgery for trichiasis, antibiotics for infection, facial cleanliness (hygiene promotion) and environmental improvements, to reduce transmission of the organism. Each of these components tackles the pathway to blindness at different stages.

\subsection{Active trachoma}

Antibiotics for treatment of active trachoma may be given locally or systematically, but topical treatment is preferred because:It is cheaper, there is no risk of systemic side-effects, and Local antibiotics are also effective against bacterial conjunctivitis which may be associated with trachoma.

The following topical and systemic therapy regimes have been recommended:

1. Topical therapy regimes. It is best for individual cases. It consist of 1 percent tetracycline or 1 percent erythromycin eye ointment 4 times a day for 6 weeks or 20 percent sulfacetamide eye drops three times a day along with 1 percent tetracycline eye ointment at bed time for 6 weeks. 
2. Systemic therapy regimes. Tetracycline or erythromycin $250 \mathrm{mg}$ orally, four times a day for 3-4 weeks or doxycycline 100mg orally twice daily for 3-4 weeks or single dose of $1 \mathrm{gm}$ azithromycin has also been reported to be equally effective in treating trachoma.

3. Combined topical and systemic therapy regime. It is preferred when the ocular infection is severe (TI) or when there is associated genital infection. It includes: (i) 1 percent tetracycline or erythromycin eye ointment 4 times a day for 6 weeks, and (ii) tetracycline or erythromycin $250 \mathrm{mg}$ orally 4 times a day for 2 weeks.

It is increasingly appreciated that there can be a major mismatch between the signs of active trachoma and the detection of chlamydial infection (Relationship between clinical signs and infection). This is a particular problem for control programmes in determining who should be offered antibiotic treatment; if only those with signs of trachoma are given antibiotic, many infected individuals with significant loads of infection would be left untreated. The WHO currently recommends that mass community-wide treatment should be used.

1. Determine the district-level prevalence of TF in 1-9-year-old children

a. If this is $10 \%$ or more, conduct mass treatment with antibiotic throughout the district

b. If this is less than $10 \%$, conduct assessment at the community level in areas of known disease

2. If assessment at the community level is undertaken

a. in communities in which the prevalence of TF in 1-9-year-old children is $10 \%$ or more, conduct mass treatment with antibiotic

b. in communities in which the prevalence of TF in 1-9-year-old children is $5 \%$ or more, but less than $10 \%$, targeted treatment should be considered

In communities in which the prevalence of TF in 1-9-year-old children is less than 5\%, antibiotic distribution is not recommended

\subsection{The sequelae}

1. Concretions should be removed with a hypodermic needle.

2. Trichiasis may be treated by epilation, electrolysis or cryolysis

3. Entropion should be corrected surgically.

4. Xerosis should be treated by artificial tears.

\subsection{Prophylaxis}

Since immunity is very poor and short lived, reinfections and recurrences are likely.

Thus following prophylactic measures may be helpful against reinfection of trachoma.

1. Hygienic measures: 
Transmission of trachoma is closely associated with personal hygiene and environmental sanitation. Facial cleanliness and environmental improvements the F\&E components of the SAFE strategy are primarily targeting the transmission of $\mathrm{C}$. trachomatis between individuals. Numerous epidemiological studies have found an association between dirty faces and active trachoma in children

Eye-seeking flies are a common feature of many trachoma endemic communities and have long been considered a potential vector. Chlamydia trachomatis was found (by PCR) on 15\% of flies caught leaving faces of children in a study from Ethiopia.

Many trachoma control programmes actively advocate for general improvements in water supply (for face washing) and sanitation (to suppress fly populations). This drive has fortunately coincided with the setting of the United Nations' Millennium Development Goals (MDG). The target for the seventh MDG is to halve the number of people without safe water and basic sanitation by 2015

2. Early treatment of conjunctivitis:

Every case of conjunctivitis should be treated as early as possible to reduce transmission of disease.

3. Blanket antibiotic therapy (intermittent treatment).

WHO has recommended this regime to be carried out in endemic areas to minimize the intensity and severity of trachoma. The regime is to apply 1 percent tetracycline eye ointment twice daily for 5 days in a month for 6 months.

The future of trachoma control

In previously endemic countries in Europe and elsewhere, trachoma declined in the face of general improvements in living conditions and health. Such changes are beginning to happen in some parts of currently endemic countries. However, for many communities it may take many decades for general improvements in living standards to happen and to have an impact on trachoma. Therefore, it is necessary to pro-actively implement the SAFE strategy as the best validated approach to control this blinding disease. The limited published data on the impact of implementing the SAFE strategy indicate that even in some of the most highly endemic regions, such as South Sudan, significant reductions in the prevalence of active disease can be achieved.

4. The development of an efficacious vaccine:

Vaccine for C. trachomatis that would prevent and resolve infection has been slow largely because of the intracellular nature of Chlamydia and lack of ability to genetically transform the organism. However, recent advances in the field have identified some requirements for vaccine design. It is now generally accepted that MOMP, possibly with other antigens, would be important for a vaccine. However, because of the diversity of MOMP sequences that define different $\mathrm{C}$. trachomatis strains, more than one MOMP would be required. The immune response that must be induced comprises mucosal sIgA antibody and systemic antigen-specific CD 4 TH1 lymphocyte responses. Protection of mice against challenge with 
MoPn has been partially successful using vaccine strategies that include conformationally intact MOMP, naked DNA constructs of ompA and intact, nonviable organisms carried by dendritic cells.175 Other attempts at vaccination were less successful and included recombinant poliovirus or Salmonella expressing MOMP, denatured MOMP, or MOMP peptides (summarized in Brunham176). It is likely that a composite vaccine that includes intact MOMP, as well as naked DNA representing ompA from various strains, may be required to stimulate appropriate B- and T-cell responses, respectively. It may also be that only a few MOMP and ompA DNA strain sequences or only specific conserved sequences from MOMP and ompA are required to elicit a protective immune response. The development of the ideal vaccine remains a significant challenge.

\section{Adult inclusion conjunctivitis}

Chlamydia sexually transmitted diseases account for more than 500 million cases worldwide; but the exact prevalence of adult inclusion conjunctivitis is unknown because infection is usually self-limited and does not always reach medical attention. The primary source of infection is from cervicitis in females and urethritis in males. The spread is from "hand -to genital tract -to eye" (during sexual activity). The incubation period is considered to be approximately 5 to 19 days. Persons between the ages of 15 and 30 years are at highest risk for adult inclusion conjunctivitis.

\subsection{Clinical features}

Symptoms include:

Foreign body sensation.

Mild photophobia and

Mucopurulent discharges from the eyes.

Signs are:

Conjunctival hyperaemia more marked in the fornices.

Acute follicular hypertrophy predominantly in the lower palpebral conjunctiva

Superficial keratitis in upper hemisphere of the cornea.

Superior micropannus may also occur.

Pre-auricular lymphadenopathy is common finding.

Clinical course: the course of the disease is benign; but often evolves into the chronic follicular conjunctivitis. 


\subsection{Differential diagnosis}

Adults develop a follicular conjunctivitis that can be indistinguishable from that of trachoma. The follicles may be present on both the lower conjunctiva and upper tarsus. The onset is usually acute with preauricular lymphadenopathy on the involved side and a serosanguineous to mucopurulent discharge. After 2 weeks of infection, corneal involvement is more prominent and includes keratitis, subepithelial opacities, and infiltrates that are marginal and/or central. Occasionally there is mild scarring and corneal vascularization referred to as micropannus, but these are late findings, usually among cases that have not been treated.

Otitis media is a common complication of chlamydial conjunctivitis. Although there can be prompt resolution of the disease, In addition there can be a genital tract disease (which failure to treat) resulting in the recurrence of the conjunctivitis.

Inclusion conjunctivitis is caused by serotypes $\mathrm{D}$ to $\mathrm{K}$ of Chlamydia tachomatis. The LGV strains (L1, L2, and L3) of C. trachomatis are responsible for a much more severe ocular disease referred to as Parinaud's oculoglandular syndrome. This syndrome is comprised of an inflammatory conjunctival response with severe lymphadenopathy involving the preauricular, cervical, and submandibular nodes. The LGV serovars are uncommon in developed countries with few reports in the literature but are very common in tropical and subtropical developing countries. Occasionally keratoconjunctivitis resulting from L2 has been reported as a consequence of laboratory accidents.

\subsection{Treatment}

The best form of treatment for adult inclusion conjunctivitis is to prevent chlamydial sexually transmitted diseases (STDs). Unfortunately, most chlamydial STDs are asymptomatic for males (approximately 40\%) and females (approximately 70\%) and usually go undetected because routine diagnostic screening for $\mathrm{C}$. trachomatis is not performed. Thus, it is important to recognize adult inclusion conjunctivitis that is caused by $\mathrm{C}$. trachomatis and treat both the ocular and genital tract disease. Because chlamydial STDs cannot be resolved by topical ocular antibiotics, systemic therapy is recommended. Most cases infected with non-LGV serovars will respond to oral tetracycline250mg four times a day for 3-4 weeks; Doxycycline100mg twice a day for 1-2 weeks or 200mg weekly for 3 weeks, or erythromycin 250mg four times a day for 3-4 weeks; when tetracycline is contraindicated as in pregnant and lactating females.

For LGV, the best treatment regimen for inclusion conjunctivitis caused by $\mathrm{C}$. psittaci and C. pneumoniae is unknown, although 6 weeks of oral antibiotics has been successful in some cases for complete eradication.

\subsection{Prophylaxis}

Patient's sexual partner should be examined and treated.

Improvement in personal hygiene and regular chlorination of swimming pool decrease the spread of disease. 


\section{Neonatal inclusion conjunctivitis}

Approximately $5 \%$ of pregnant women have C. trachomatis infection of the cervix.which if left untreated has $50 \%$ chance of the infant developing conjunctivitis. Neonatal inclusion conjunctivitis is more common (about 10 times) than conjunctivitis resulting from N. gonorrhoeae. The incubation period for chlamydial conjunctivitis is 1 to 3 weeks. Earlier infection can occur if there is evidence for rupture of membranes. If left untreated, the conjunctivitis can persist for 3 to 12 months. C. trachomatis is the leading cause of pneumonitis within the first 6 months of life. Rectal shedding of C. trachomatis does occur and is more common among infants with pneumonia. Onset of shedding does not usually occur before 6 to 12 weeks of age and can be as late as 12 months.

\subsection{Clinical features}

Conjunctivitis in the neonate is characterized by swelling of lids, a purulent discharge, and hyperemia. If without treatment, neonates are at risk for conjunctival scarring, keratitis, and superficial vascularization of the cornea. In addition, these infants if up to 6 months of age are at risk for pneumonitis.

\subsection{Treatment}

Treating pregnant women is effective in preventing infants from acquiring conjunctival infection, although retreatment may be necessary in high-risk populations. In infants, systemic treatment is recommended for the fact that topical treatment of neonatal inclusion conjunctivitis does not eradicate nasopharyngeal carriage, which can result in pneumonia or recurrent ocular infection. In addition, mothers should be treated for genital tract infection to prevent recurrence of chlamydial conjunctivitis in the neonate.

\section{Author details}

Udo Ubani

Dept. of Optometry, Abia State University, Uturu, Nigeria

\section{References}

[1] Alemayehu W, Melese M, Bejiga A et al. (2004) Surgery for trichiasis by ophthalmologists versus integrated eye care workers: a randomized trial. Ophthalmology, 111, 578-584. 
[2] Batt SL, Charalambous BM, Solomon AW et al. (2003) Impact of azithromycin administration for trachoma control on the carriage of antibiotic-resistant Streptococcus pneumoniae.Antimicrob Agents Chemother, 47, 2765-2769.

[3] Berhane Y, Worku A, Bejiga A (2006) National Survey on Blindness, Low Vision and Trachoma in Ethiopia. Federal Ministry of Health of Ethiopia.

[4] Bowman RJ, Soma OS, Alexander N et al. (2000) Should trichiasis surgery be offered in the village? A community randomised trial of village vs. health centre-based surgery. Trop Med Int Health, 5, 528-533.

[5] Bowman RJ, Sillah A, Dehn C et al. (2000) Operational comparison of single-dose azithromycin and topical tetracycline for trachoma. Invest Ophthalmol Vis Sci, 41, 40744079 .

[6] Burton MJ, Holland MJ, Faal N et al. (2003) Which members of a community need antibiotics to control trachoma? Conjunctival chlamydia trachomatis infection load in Gambian villages. Invest Ophthalmol Vis Sci, 44, 4215-4222.

[7] Burton MJ, Bailey RL, Jeffries D et al. (2004) Cytokine and fibrogenic gene expression in the conjunctivas of subjects from a Gambian community where trachoma is endemic. Infect Immun, 72, 7352-7356.

[8] Burton MJ, Holland MJ, Makalo P et al. (2005) Re-emergence of Chlamydia trachomatis infection after mass antibiotic treatment of a trachoma-endemic Gambian community: a longitudinal study. Lancet, 365, 1321-1328.

[9] Burton MJ, Kinteh F, Jallow O et al. (2005) A randomised controlled trial of azithromycin following surgery for trachomatous trichiasis in the Gambia. Br J Ophthalmol, $89,1282-1288$.

[10] Burton MJ, Holland MJ, Jeffries D et al. (2006) Conjunctival chlamydial 16S ribosomal RNA expression in trachoma: is chlamydial metabolic activity required for disease to develop? Clin Infect Dis, 42, 463-470.

[11] Burton MJ, Bowman RJ, Faal H et al. (2006) The long-term natural history of trachomatous trichiasis in the gambia. Invest Ophthalmol Vis Sci, 47, 847-852.

[12] Centers for Disease Control and Prevention. (2001) Sexually transmitted diseases treatment guidelines,. MMWR Morb Mortal Wkly Rep, 55 (RR-11), 1-94.

[13] Dean D, Suchland R, Stamm W. (2000) Evidence for long-term cervical persistence of Chlamydia trachomatis by omp1 genotyping. J Infect Dis;182:909

[14] Dean D, Powers VC. (2001) Persistent Chlamydia trachomatis infections resist apoptotic stimuli. Infect Immun;69:2442

[15] Emerson PM, Bailey RL, Mahdi OS et al. (2000) Transmission ecology of the fly Musca sorbens, a putative vector of trachoma. Trans R Soc Trop Med Hyg;94:28 
[16] Emerson PM, Cairncross S, Bailey RL et al. (2000) Review of the evidence base for the ' $\mathrm{F}$ ' and ' $\mathrm{E}$ ' components of the SAFE strategy for trachoma control. Trop Med Int Health, 5,515-527.

[17] Emerson PM, Lindsay SW, Alexander N et al. (2004) Role of flies and provision of latrines in trachoma control: cluster-randomised controlled trial. Lancet, 363, 10931098.

[18] Gray RH, Wabwire-Mangen F, Kigozi G et al. (2001) Randomized trial of presumptive sexually transmitted disease therapy during pregnancy in Rakai, Uganda. Am J Obstet Gynecol, 185, 1209-1217.

[19] Hessel T, Dhital SP, Plank R et al. (2001) Immune response to chlamydial 60-kilodalton heat shock protein in tears from Nepali trachoma patients. Infect Immun;69:4996

[20] Holm SO, Jha HC, Bhatta RC et al. (2001)Comparison of two azithromycin distribution strategies for controlling trachoma in Nepal. Bull World Health Organ;79:194

[21] Hsia RC, Ahmed I, Batteiger B et al. (2000) Differential immune response to polymorphic membrane proteins in STD patients. Fourth Meeting of the European Society for Chlamydia Research. Helsinki: Societa Editrice Esculapio.:219

[22] Hsieh YH, Bobo LD, Quinn TC et al. (2001) Determinants of trachoma endemicity using Chlamydia trachomatis ompA DNA sequencing. Microbes Infect;3:447

[23] Jones RB, Batteiger BE (2000). Chlamydia Trachomatis. In Mandell GL, Bennett GE, DolinR (eds): Douglas and Bennett's Principles and Practice of Infectious Diseases, Ed 5. Philadelphia: Churchill Livingstone.:1986-1989

[24] Khandekar R, Mohammed AJ, Courtright P (2001) Recurrence of trichiasis: a longterm follow-up study in the Sultanate of Oman. Ophthalmic Epidemiol, 8, 155-161.

[25] Mabey D, Fraser-Hurt N (2001). Trachoma. BMJ;323:218

[26] Michel CE, Solomon AW, Magbanua JP et al. (2006) Field evaluation of a rapid pointof-care assay for targeting antibiotic treatment for trachoma control: a comparative study. Lancet, 367, 1585-1590.

[27] Miller K, Pakpour N, Yi E et al. (2004) Pesky trachoma suspect finally caught. Br J Ophthalmol, 88, 750-751.

[28] Millman KL, Tavare S, Dean D. (2001) Recombination in the ompA gene but not the omcB gene of Chlamydia contributes to serovar-specific differences in tissue tropism, immune surveillance, and persistence of the organism. J Bacteriol;183:5997

[29] Ngondi J, Onsarigo A, Matthews F et al. (2006) Effect of 3 years of SAFE (surgery, antibiotics, facial cleanliness, and environmental change) strategy for trachoma control in southern Sudan: a cross-sectional study. Lancet, 368, 589-595. 
[30] Natividad A, Wilson J, Koch O et al. (2005) Risk of trachomatous scarring and trichiasis in Gambians varies with SNP haplotypes at the interferon-gamma and interleukin-10 loci. Genes Immun, 6, 332-340.

[31] Natividad A, Cooke G, Holland M et al. (2006) A coding polymorphism in Matrix Metalloproteinase 9 reduces risk of scarring sequelae of ocular Chlamydia trachomatis infection. BMC Med Genet, 7, 40.

[32] Natividad A, Hanchard N, Holland MJ et al. (2007) Genetic variation at the TNF locus and the risk of severe sequelae of ocular Chlamydia trachomatis infection in Gambians. Genes Immun, doi:10.1038/sj.gene.6364384.

[33] Polack S, Brooker S, Kuper H et al. (2005) Mapping the global distribution of trachoma. Bull World Health Organ, 83, 913-919.

[34] Rabiu MM, Abiose A. (2001) Magnitude of trachoma and barriers to uptake of lid surgery in a rural community of northern Nigeria. Ophthalmic Epidemiol;8:181

[35] Resnikoff S, Pascolini D, Etya'ale D et al. (2004) Global data on visual impairment in the year 2002. Bull World Health Organ, 82, 844-851.

[36] Rottenberg ME, Gigliotti-Rothfuchs A, Wigzell H (2002) The role of IFN-gamma in the outcome of chlamydial infection. Curr Opin Immunol, 14, 444-451.

[37] Shirai M, Hirakawa H, Kimoto M et al. (2000) Comparison of whole genome sequences of Chlamydia pneumoniae J138 from Japan and CWL029 from USA. Nucleic Acids Res;28:2311

[38] Solomon AW, Holland MJ, Burton MJ et al. (2003) Strategies for control of trachoma: observational study with quantitative PCR. Lancet, 362, 198-204.

[39] Solomon AW, Peeling RW, Foster A et al. (2004) Diagnosis and assessment of trachoma. Clin Microbiol Rev, 17, 982-1011.

[40] Solomon AW, Holland MJ, Alexander ND et al. (2004) Mass treatment with singledose azithromycin for trachoma. N Engl J Med, 351, 1962-1971.

[41] Suchland RJ, Rockey DD, Bannantine JP et al. (2000) Isolates of Chlamydia trachomatis that occupy nonfusogenic inclusions lack IncA, a protein localized to the inclusion membrane. Infect Immun;68:360

[42] West SK, Munoz B, Mkocha H et al. (2001) Progression of active trachoma to scarring in a cohort of Tanzanian children. Ophthalmic Epidemiol, 8, 137-144.

[43] West SK, Munoz B, Mkocha H et al. (2005) Infection with Chlamydia trachomatis after mass treatment of a trachoma hyperendemic community in Tanzania: a longitudinal study. Lancet, 366, 1296-1300. 
[44] West SK, West ES, Alemayehu W et al. (2006) Single-dose azithromycin prevents trichiasis recurrence following surgery: randomized trial in Ethiopia. Arch Ophthalmol, 124, 309-314

[45] West SK, Emerson PM, Mkocha $\mathrm{H}$ et al. (2006) Intensive insecticide spraying for fly control after mass antibiotic treatment for trachoma in a hyperendemic setting: a randomised trial.Lancet, 368, 596-600.

[46] Wright HR, Taylor HR (2005) Clinical examination and laboratory tests for estimation of trachoma prevalence in a remote setting: what are they really telling us? Lancet Infect Dis, 5, 313-320.

[47] Wynn TA (2004) Fibrotic disease and the $\mathrm{T}(\mathrm{H}) 1 / \mathrm{T}(\mathrm{H}) 2$ paradigm. Nat Rev Immunol, $4,583-594$. 
